\title{
CT perfusion imaging of the liver and the spleen in patients with cirrhosis: Is there a correlation between perfusion and portal venous hypertension?
}

\author{
Emina Talakić $^{1} \cdot$ Silvia Schaffellner $^{2}$ - Daniela Kniepeiss ${ }^{2} \cdot$ Helmut Mueller $^{2}$ • \\ Rudolf Stauber $^{3} \cdot$ Franz Quehenberger $^{4} \cdot$ Helmut Schoellnast ${ }^{1}$
}

Received: 31 October 2016 / Revised: 12 January 2017 / Accepted: 23 February 2017 / Published online: 20 March 2017

(C) The Author(s) 2017. This article is published with open access at Springerlink.com

\begin{abstract}
Objectives To correlate hepatic and splenic CT perfusion parameters with hepatic venous pressure gradient (HVPG) measurements in patients with cirrhosis.

Methods Twenty-one patients with cirrhosis (males, 17; females, 4 ; mean $\pm \mathrm{SD}$ age, $57 \pm 7$ years) underwent hepatic and splenic perfusion $\mathrm{CT}$ on a 320-detector row volume scanner as well as invasive measurement of HVPG. Different CT perfusion algorithms (maximum slope analysis and Patlak plot) were used to measure hepatic arterial flow (HAF), portal venous flow (PVF), hepatic perfusion index (HPI), splenic arterial flow (SAF), splenic blood volume (SBV) and splenic clearance (SCL). Hepatic and splenic perfusion parameters were correlated with HVPG, and sensitivity and specificity for detection of severe portal hypertension $(\geq 12 \mathrm{mmHg})$ were calculated.

Results The Spearman correlation coefficient was -0.53 $(\mathrm{p}<0.05)$ between SAF and HVPG, and $-0.68(\mathrm{p}<0.01)$ between HVPG and SCL. Using a cut-off value of $125 \mathrm{ml} / \mathrm{min} /$ $100 \mathrm{ml}$ for SCL, sensitivity for detection of a HVPG of $\geq 12 \mathrm{mmHg}$ was $94 \%$, and specificity $100 \%$. There was no significant correlation between hepatic perfusion parameters and HVPG.
\end{abstract}

Helmut Schoellnast

helmut.schoellnast@medunigraz.at

1 Division of General Radiology, Department of Radiology, Medical University of Graz, Auenbruggerplatz 9, Graz A-8036, Austria

2 Department of Surgery, Division of Transplantation Surgery, Medical University of Graz, Graz, Austria

3 Department of Internal Medicine, Division of Gastoenterology and Hepatology, Medical University of Graz, Auenbruggerplatz 15, 8036 Graz, Austria

4 Institute for Medical Informatics, Statistics and Documentation, Medical University of Graz, Auenbruggerplatz 2, Graz 8036, Austria
Conclusion CT perfusion in patients with cirrhosis showed a strong correlation between SCL and HVPG and may be used for detection of severe portal hypertension.

Key points

- SAF and SCL are statistically significantly correlated with HVPG

- SCL showed stronger correlation with HVPG than SAF

- $125 \mathrm{ml} / \mathrm{min} / 100 \mathrm{ml}$ SCL-cut-off yielded $94 \%$ sensitivity, $100 \%$ specificity for severe $P H$

- HAF, PVF and HPI showed no statistically significant correlation with $H V P G$

Keywords CT-perfusion · Liver · Spleen · Cirrhosis · Portal hypertension

$\begin{array}{ll}\text { Abbreviations and acronyms } \\ \text { HAF } & \text { Hepatic arterial flow }(\mathrm{ml} / \mathrm{min} / 100 \mathrm{ml}) \\ \text { HPI } & \text { Hepatic perfusion index }(\%) \\ \text { HVPG } & \text { Hepatic venous pressure gradient }(\mathrm{mmHg}) \\ \text { PH } & \text { Portal hypertension } \\ \text { PVF } & \text { Portal venous flow }(\mathrm{ml} / \mathrm{min} / 100 \mathrm{ml}) \\ \text { SAF } & \text { Splenic arterial flow }(\mathrm{ml} / \mathrm{min} / 100 \mathrm{ml}) \\ \text { SBV } & \text { Splenic blood volume }(\mathrm{ml} / 100 \mathrm{ml}) \\ \text { SCL } & \text { Splenic clearance }(\mathrm{ml} / \mathrm{min} / 100 \mathrm{ml})\end{array}$

\section{Introduction}

Portal hypertension $(\mathrm{PH})$ is defined as an increase in the pressure in the portal vein and its territory, and is one of the main causes of severe complications and death in patients with cirrhosis and, therefore, the main prognostic factor in cirrhosis [1]. In normal fasted subjects at rest and in the supine position, portal pressure ranges from 7 to $12 \mathrm{mmHg}$ [2]. The direct 
measurement of portal pressure is a markedly invasive technique that is no longer performed in patients with cirrhosis; the indirect, less invasive technique of measuring the hepatic venous pressure gradient (HVPG) is used as a standard of reference to estimate the severity of PH in cirrhosis. In healthy subjects, the pressure gradient between the portal vein territory and the vena cava territory ranges from 1 to $4 \mathrm{mmHg}$ [3]. $\mathrm{PH}$ is considered moderate when the HVPG ranges from 5 to $10 \mathrm{mmHg}$ and severe when the HVPG is greater than $10-12 \mathrm{mmHg}$. [1, 4, 5]. An HVPG threshold of $10 \mathrm{mmHg}$ is termed clinically significant $\mathrm{PH}$ as it predicts the risk of complications such as formation of oesophageal varices and development of hepatocellular carcinoma [6-8]. An HVPG $>12 \mathrm{mmHg}$ is associated with the risk of variceal bleeding [4] and an HVPG $>16 \mathrm{mmHg}$ correlates with increased mortality $[9,10]$.

Although HVPG measurement is a safe technique with only minor complications in $<1 \%$ of patients [1], noninvasive techniques for HVPG assessment would be more favourable than invasive techniques. Computed tomography perfusion imaging (CTP) is a functional imaging technique that has been proven to allow quantification of the hepatic blood circulation in patients with diffuse liver disease [11-13]. As the hepatic blood circulation is commonly affected by chronic liver disease, mainly due to parenchymal remodeling resulting in an increased vascular resistance of the liver, CTP may be a valuable tool for noninvasive assessment of PH. In addition, splenic blood circulation assessed by CTP may be influenced by PH.

The aim of this study was to evaluate whether a correlation exists between hepatic and splenic perfusion parameters assessed by CTP and HVPG assessed by hepatic venous catherization.

\section{Materials and methods}

\section{Patient population}

This prospective study was approved by the Institutional Ethics Committee and informed consent was obtained from all patients. Patients who underwent HVPG measurement for evaluation of liver transplantation underwent CTP of the liver and the spleen. Inclusion criteria were: (a) HVPG measurement for suspected portal hypertension, and (b) age 18 years or older. Exclusion criteria were: (a) renal impairment (estimated glomerular filtration rate (eGFR) $<45 \mathrm{ml} / \mathrm{min} / 1,73 \mathrm{qm}$ ), (b) other contraindications to the application of iodinated contrast media, and (c) pregnancy.

Twenty-one patients (18 male, three female; mean $\pm \mathrm{SD}$ age, $57 \pm 7$ years; range, $41-69$ years) were included in the study. Twenty patients had cirrhosis and one patient had neuroendocrine liver metastases. Of the patients with cirrhosis, Child-Pugh classification was A in two patients, B in eight patients and $\mathrm{C}$ in eight patients. In two patients no classification was available in the medical records. Fourteen patients had alcoholic cirrhosis, three patients had cirrhosis due to hepatitis $\mathrm{C}$ and three patients had cryptogenic cirrhosis.

All patients underwent CTP of the liver and the spleen as well as HVPG measurement. The mean period $( \pm \mathrm{SD})$ between CTP and HPGV measurement was $2 \pm 2$ days (range, $0-9$ days).

\section{CTP imaging technique}

All VCTP studies were performed using a 320-detector row CT (Aquilion One, Toshiba Medical Systems, Otawara, Japan) following an overnight fast. Scanning parameters were as follows: $100 \mathrm{kV}$ tube voltage, $100 \mathrm{~mA}$ tube current, $0.5 \mathrm{~s}$ gantry rotation time and $320 \times 0.5-\mathrm{mm}$ section thickness. The detector width was $16 \mathrm{~cm}$. A low-dose pre-contrast helical scan of the abdomen during suspended respiration following inspiration was performed to locate the liver and the spleen and to plan the scan position for the CTP study. As recommended for CT-angiography, contrast dose and injection rate were adjusted to patients' body weight in our CT-perfusion protocol [14] (40-60 ml: 50-69 kg body weight, $40 \mathrm{ml}$; 70$89 \mathrm{~kg}$ body weight, $50 \mathrm{ml}$; and $>90 \mathrm{~kg}$ body weight, $60 \mathrm{ml}$ ).Iomeprol $400 \mathrm{mgI} / \mathrm{ml}$ (Iomeron®, Bracco, Milano, Italy) was injected at a flow rate of 6-8 ml/s (50-69 kg body weight, $6 \mathrm{ml} / \mathrm{s} ; 70-89 \mathrm{~kg}$ body weight, $7 \mathrm{ml} / \mathrm{s}$; and $>90 \mathrm{~kg}$ body weight, $8 \mathrm{ml} / \mathrm{s}$ ), followed by the same volume of saline solution at the same injection rate using a dual-head power injector (Ulrich Medical, Chesterfield, MO, USA). All in all 21 volume acquisitions were obtained without table movement in all patients. After acquisition of two pre-contrast volumes, eight volumes were acquired during the early period of the injection protocol with an interscan gap of $1 \mathrm{~s}$ followed by seven volume acquisitions with an interscan gap of $2 \mathrm{~s}$ and by further four volume acquisitions with an interscan gap of $4 \mathrm{~s}$. Total scan time was $80 \mathrm{~s}$. Every patient was instructed to breathe normally during the examination and to avoid deep and irregular breathing. A band compressing the upper abdomen was used to reduce liver excursions. The total doselength product (DLP) of 21 volumes was 1,031.1 mGy.cm, which is equivalent to an effective dose of $15.4 \mathrm{mSv}(\mathrm{k} 0.015)$.

\section{Image analysis}

Post-processing was performed using body perfusion software (Body Perfusion, Toshiba Medical Systems) as available on the CT equipment. First, body registration to correct motion between the dynamic volumes was performed. Following registration, the corrected volumes were loaded into the body perfusion software. 
The dual input maximum slope model [15] was used for perfusion analysis of the liver. Two methods were used with regard to the settings of the regions of interest (ROIs) in all patients. In Method I the ROIs were manually placed in the abdominal aorta, the portal vein, the spleen and normal liver parenchyma to generate respective time-density curves (TDCs). The generated TDCs represented the hepatic artery input function and the portal vein input function, respectively (Fig. 1A). The peak point of the generated TDC of the spleen is used to separate the hepatic artery circulation before the peak point and the portal vein circulation after the peak point, respectively. In Method II the break point was manually set at the crossing point of the aortal and portal venous TDC (Fig. 1B). This adapted method was performed as it has been shown that in patients with portal venous hypertension peak splenic enhancement may be delayed, which may alter perfusion measurements due to inaccurate separation of arterial and portal venous perfusion [16]. Hepatic arterial blood flow (HAF; $\mathrm{ml} / \mathrm{min} /$ $100 \mathrm{ml}$ ), portal venous blood flow (PVF; $\mathrm{ml} / \mathrm{min} /$ $100 \mathrm{ml}$ ), as well as the hepatic perfusion index (HPI; $\mathrm{HAF} /(\mathrm{HAF}+\mathrm{PVF}) ; \%$ ) were calculated for all liver segments and the results were averaged for the whole liver.

The single input maximum slope and the Patlak plot model [15] were used for perfusion analysis of the spleen. ROIs were manually placed in the abdominal aorta and the spleen to generate respective TDCs. With the use of the single input maximum slope model the splenic arterial blood flow (SAF; $\mathrm{ml} / \mathrm{min} / 100 \mathrm{ml}$ ) and with the Patlak plot model, the splenic blood volume $(\mathrm{SBV} ; \mathrm{ml} / 100 \mathrm{ml}$ ) and the splenic clearance (SCL; ml/ $\mathrm{min} / 100 \mathrm{ml}$ ), which is the total flux from the intravascular space to the extravascular space, were calculated.

In addition to CT-perfusion parameters, morphological features such as presence of perihepatic ascites, portosystemic collateral vessels and portal thrombosis were qualitatively assessed and spleen volume was quantitatively assessed using semiautomatic volumetric software (Vitrea, Toshiba Medical Systems).

\section{HVPG measurement}

HVPG measurements were carried out according to established standards [17] following an overnight fast. Under ultrasound guidance, the right internal jugular vein was cannulated and a 7-French balloon catheter (Boston Scientific) was guided into the right hepatic vein for the measurement of wedged and free hepatic venous pressures. HVPG was calculated from the difference between wedged hepatic venous pressure (WHVP) and free hepatic venous pressure, and the mean of triplicate measurements was computed.

\section{Statistical analysis}

The data were descriptively reviewed. The significances of differences in medians between Method I and Method II were analysed using Wilcoxon's test. Spearman's test was used for correlation of the perfusion parameters with the HVPG. Comparison of the parameters between the severe $(\geq 12 \mathrm{mmHg}$ ) and moderate $(<12 \mathrm{mmHg})$ portal hypertension groups and between patients with and without portal thrombosis was assessed using the Mann-Whitney test. Receiver operating characteristics (ROC) analysis was performed to calculate cut-off values for differentiation between moderate and severe portal hypertension. The significance of distribution of morphological features between the two groups was tested using the Chi-square test. Statistical analysis was performed with commercially available software (IBM SPSS Statistics, Version 23, SPSS Inc., Chicago, IL, USA). A Pvalue $<0.05$ was considered statistically significant.

\section{Results}

Details on hepatic and splenic perfusion values and HVPG are listed in Table 1.

There was no statistically significant difference between Method I and Method II in HAF, whereas PVF and HPI were statistically significant different $(\mathrm{p}<0.05)$. The mean difference in medians $( \pm \mathrm{SD})$ was $1.0 \pm 2.3 \mathrm{ml} / \mathrm{min} / \mathrm{ml}$ for HAF (range, $0-9.1 \mathrm{ml} / \mathrm{min} / 100 \mathrm{ml}$ ), $22.6 \pm 43.3 \mathrm{ml} / \mathrm{min} / 100 \mathrm{ml}$ for PVF (range, $0-146.1 \mathrm{ml} / \mathrm{min} / 100 \mathrm{ml}$ ) and $9.1 \pm 14.4 \mathrm{ml} / \mathrm{min} /$ $100 \mathrm{ml}$ for HPI (range, $0-41.6 \mathrm{ml} / \mathrm{min} / 100 \mathrm{ml}$ ).

For Method I as well as for Method II no statistically significant correlation was found between HAF, PVF, HPI and HVPG. On the contrary, SAF and SCL showed a statistically significant negative correlation with the HVPG, whereas SBV showed no correlation. The Spearman correlation coefficient was $-0.53(\mathrm{p}<0.05)$ for SAF and $-0.68(\mathrm{p}<0.01)$ for SCL. Figure 2 shows scatter plots for SAF and HVPG and for SCL and HVPG, respectively. There was no statistically significant correlation between splenic volume and HVPG.

Five patients had moderate (HVPG $<12 \mathrm{mmHg}$ ) and 16 patients had severe (HVPG $\geq 12 \mathrm{mmHg}$ ) portal hypertension. The median HVPG was $7 \mathrm{mmHg}$ (interquartile range (IQR), $8 \mathrm{mmHg}$; range, $2-11 \mathrm{mmHg}$ ) in the moderate portal hypertension group and $16 \mathrm{mmHg}$ (IQR, $8 \mathrm{mmHg}$; range, $12-28 \mathrm{mmHg}$ ) in the severe portal hypertension group. Child-Pugh classification was $\mathrm{A}$ in one patient, $\mathrm{B}$ in two patients and $\mathrm{C}$ in one patient in the moderate portal hypertension group. One patient of this group had no cirrhosis. In the severe portal hypertension group Child-Pugh classification was A in one patient, B in six patients and $\mathrm{C}$ in seven patients. In two patients of this group Child-Pugh classification was not available. 
a

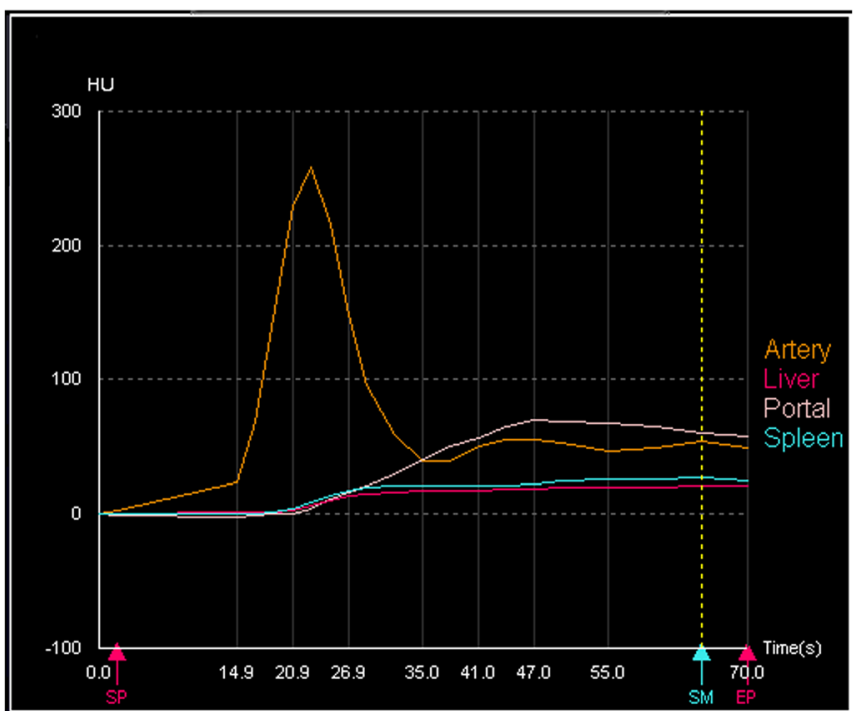

C

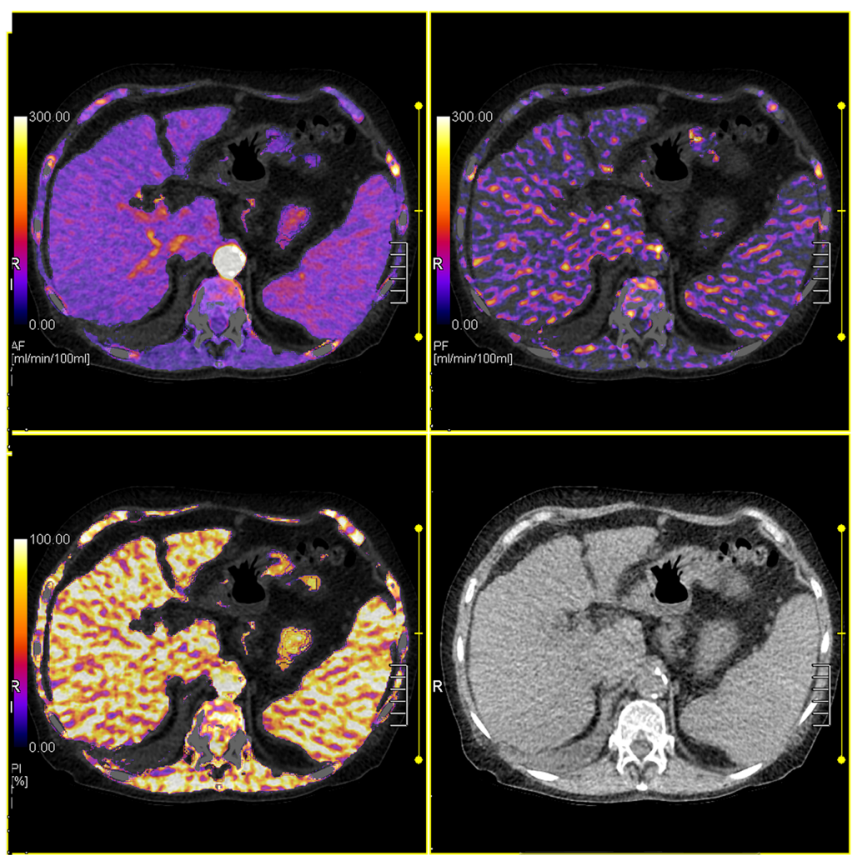

Fig. 1 (A-D) Dual input maximum slope model of the liver yielding time-density-curves (TDC) for the aorta, the portal vein, the spleen and the liver $(\mathbf{A}, \mathbf{B})$ and parametric images $(\mathbf{C}, \mathbf{D})$ for hepatic arterial flow (HAF; left top), portal venous flow (PVF; right top) and hepatic perfusion index (HPI; left bottom) as well as corresponding pre-contrast grey-scale images (right bottom). The peak point of the TDC of the spleen is used to separate the hepatic artery circulation before the peak point and the portal vein circulation after the peak point, respectively. In patients with cirrhosis, the TDC of the liver may be flattened with a delayed peak

Body weight did not significantly differ between the two groups. Median body weight was $64 \mathrm{~kg}$ (range, 60$109 \mathrm{~kg}$ ) in the moderate portal hypertension group and $81.5 \mathrm{~kg}$ (range, $60-110 \mathrm{~kg}$ ) in the severe portal hypertension group.

\section{b}

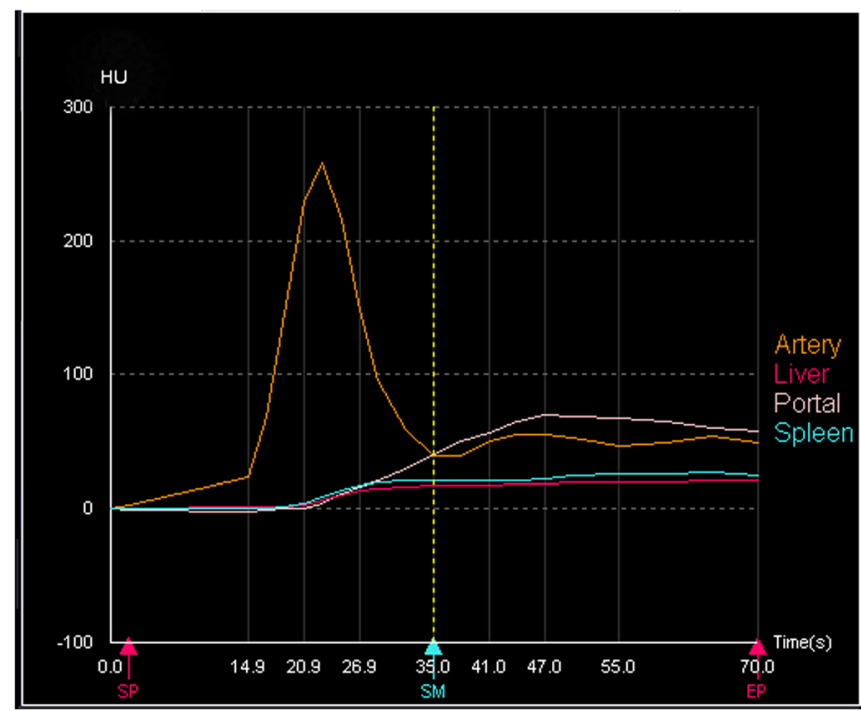

d

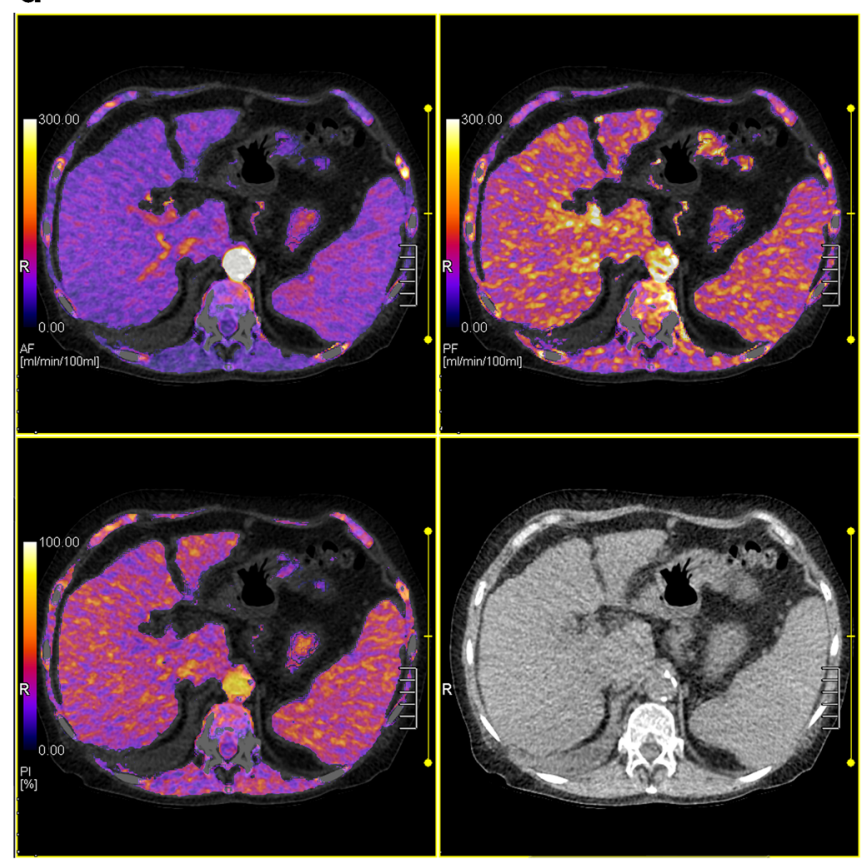

(A). Perfusion calculation with this setting led to very low PVF and very high HPI (C). Manual adaptation with placement of the breakpoint at the crossing point of the aortal and portal venous TDC $(\mathbf{B})$ led to significantly higher PVF and lower HPI (D). HAF, PVF and HPI in this patient were $68.5 \mathrm{ml} / \mathrm{min} / 100 \mathrm{ml}, 51.2 \mathrm{ml} / \mathrm{min} / 100 \mathrm{ml}$ and $61.4 \%$ for the standard setting (A, C), and $71.4 \mathrm{ml} / \mathrm{min} / 100 \mathrm{ml}, 126.5 \mathrm{ml} / \mathrm{min} / 100 \mathrm{ml}$ and $38.1 \%$ for the manual adapted setting (B, D). Severe portal hypertension (HVPG $22 \mathrm{mmHg}$ ) may contribute to the flat shape of the splenic TDC in this patient. $S P$ start point, $E P$ end point, $S M$ spleen maximum

SCL was statistically significantly different in patients with moderate and those with severe portal hypertension $(\mathrm{P}<0.01)$, whereas SAF was not different between the two groups. Median SCL in patients with moderate portal hypertension was $133.2 \mathrm{ml} / \mathrm{min} / 100 \mathrm{ml}$ (IQR, 20.7; range, $127.8-160.8 \mathrm{ml} /$ 
Table 1 Details of hepatic and splenic perfusion parameters, splenic volume and HVPG

\begin{tabular}{|c|c|c|c|c|c|}
\hline & & Median & IQR & Min & Max \\
\hline \multirow[t]{8}{*}{ Liver } & Method I & & & & \\
\hline & $\mathrm{HAF}(\mathrm{ml} / \mathrm{min} / 100 \mathrm{ml})$ & 56.7 & 26.2 & 26.4 & 123.9 \\
\hline & $\operatorname{PVF}(\mathrm{ml} / \mathrm{min} / 100 \mathrm{ml})$ & 118.0 & 81.9 & 31.1 & 255.8 \\
\hline & HPI $(\%)$ & 35.9 & 26.7 & 16.0 & 81.7 \\
\hline & Method II & & & & \\
\hline & $\mathrm{HAF}(\mathrm{ml} / \mathrm{min} / 100 \mathrm{ml})$ & 56.7 & 30.3 & 26.4 & 121.2 \\
\hline & $\operatorname{PVF}(\mathrm{ml} / \mathrm{min} / 100 \mathrm{ml})$ & 134.1 & 62.6 & 93.1 & 255.8 \\
\hline & HPI (\%) & 35.5 & 16.0 & 16.0 & 48.0 \\
\hline \multirow[t]{4}{*}{ Spleen } & $\mathrm{SAF}(\mathrm{ml} / \mathrm{min} / 100 \mathrm{ml})$ & 107.8 & 56.4 & 67.2 & 209.2 \\
\hline & $\mathrm{SBV}(\mathrm{ml} / 100 \mathrm{ml})$ & 1.5 & 2.3 & 0.4 & 39.9 \\
\hline & $\mathrm{SCL}(\mathrm{ml} / \mathrm{min} / 100 \mathrm{ml})$ & 86,4 & 69 & 23.4 & 160,8 \\
\hline & $\mathrm{V}(\mathrm{ml})$ & 577.1 & 435.0 & 149.4 & 1274.1 \\
\hline HVPG (mmHg) & & 14 & 9 & 2 & 28 \\
\hline
\end{tabular}

$H A F$ hepatic arterial flow, $P V F$ portal venous flow, $H P I$ perfusion index, $S A F$ splenic arterial flow, $S B V$ splenic blood volume, $S C L$ splenic clearance, $V$ volume, $H V P G$ hepatic venous pressure gradient $\mathrm{min} / 100 \mathrm{ml}$ ); in patients with severe portal hypertension median $\mathrm{SCL}$ was $74.1 \mathrm{ml} / \mathrm{min} / 100 \mathrm{ml}$ (IQR, 51.8, range, 23.4-138.6 ml/ $\min / 100 \mathrm{ml})$.

ROC analysis of SCL for differentiation between moderate and severe portal hypertension showed an area under the curve of 0.96 with a standard error of 0.04 (95\% confidence interval (CI), 0.88-1) (Fig. 3). Using a cut-off value of $125 \mathrm{ml} /$ $\mathrm{min} / 100 \mathrm{ml}$ for SCL, sensitivity for detection of severe portal hypertension was $94 \%$ with a specificity of $100 \%$.

Perihepatic ascites was present in three patients with moderate portal hypertension and in seven patients with severe portal hypertension; portal systemic varicose collateral vessels were present in three patients with moderate portal hypertension and in six patients with severe portal hypertension; and portal vein thrombosis was present in none of the patients with moderate portal hypertension and in four patients with severe portal hypertension. The distribution of these morphological parameters between patients with moderate and those with severe portal hypertension was not statistically significant. There was no statistically significant difference in splenic perfusion parameters and in HVPG between patients with and those without portal thrombosis. The median SAF was $93 \mathrm{ml} / \mathrm{min} / 100 \mathrm{ml}$ (IQR, 65.6; range, 77.3-163 ml/min/ $100 \mathrm{ml}$ ) in patients with portal thrombosis and $109.8 \mathrm{ml} /$ $\mathrm{min} / 100 \mathrm{ml}$ (IQR, 56.2; range, 67.2-209.2 ml/min/100 ml) in patients without portal thrombosis. The corresponding values were $1.9 \mathrm{ml} / 100 \mathrm{ml}$ (IQR, 1.4; range $1.2-2.7 \mathrm{ml} /$ $100 \mathrm{ml})$ and $1.5 \mathrm{ml} / 100 \mathrm{ml}(\mathrm{IQR}, 3.8$; range, $0.4-39.9 \mathrm{ml} /$

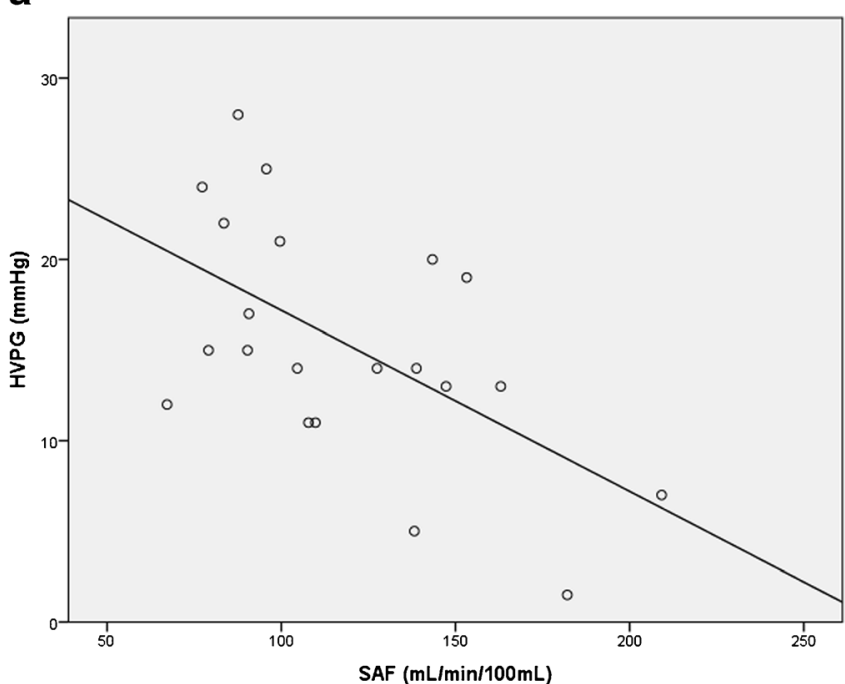

b

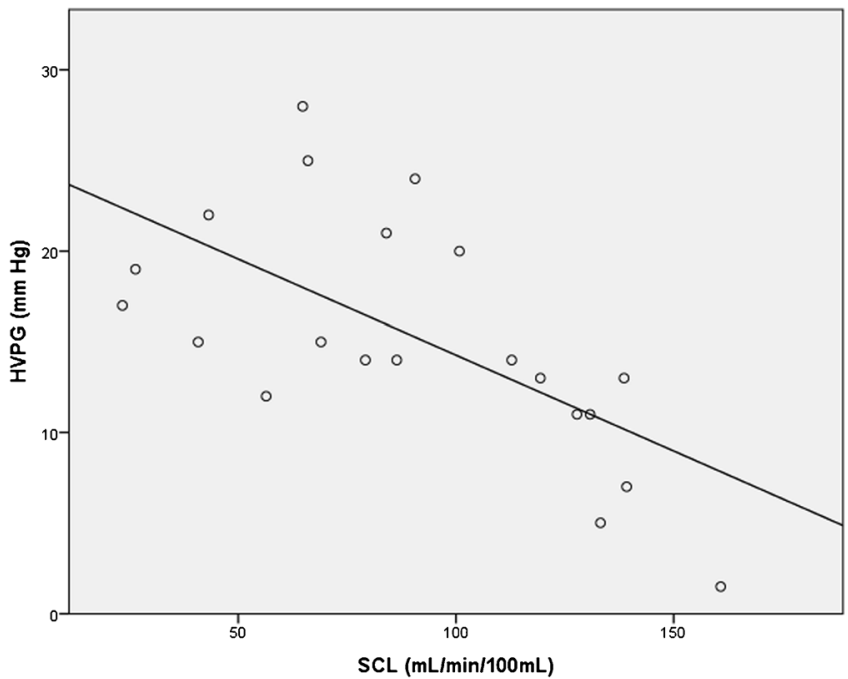

Fig. 2 (A, B) Correlation between splenic arterial flow (SAF) and hepatovenous pressure gradient (HVPG) (A) and splenic clearance (SCL) and HVPG (B) showing a moderate negative correlation $(r=0.53 . \mathrm{p}<0.05)$ for SAF and strong negative correlation $(\mathrm{r}=0.68, \mathrm{p}<0.01)$ for $\mathrm{SCL}$, respectively 


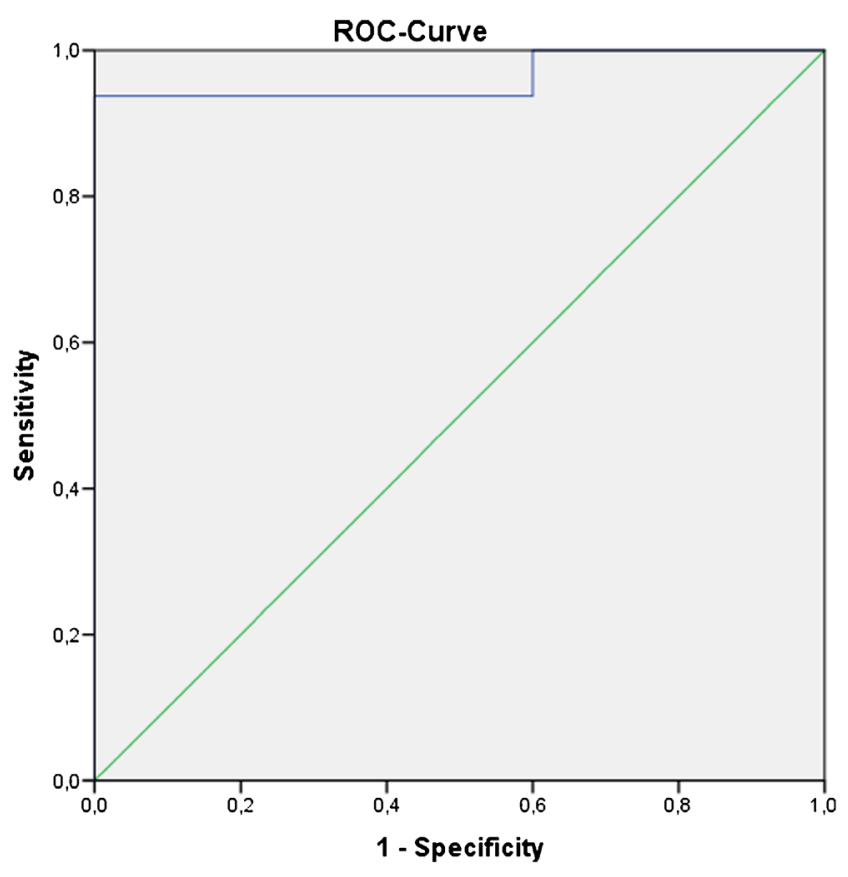

Fig. 3 Receiver operating characteristic (ROC) curves of for differentiation between moderate and severe portal hypertension $(\mathrm{PH})$ splenic clearance (SCL) for identifying severe PH. Area under the ROC was 0.96 (standard error, 0.04 ; 95\% CI, 0.88-1)

$100 \mathrm{ml}$ ) for $\mathrm{SBV}$, and $79.8 \mathrm{ml} / \mathrm{min} / 100 \mathrm{ml}$ (IQR, 59.8; range, $66-138.6 \mathrm{ml} / \mathrm{min} / 100 \mathrm{ml}$ ) and $86.4 \mathrm{ml} / \mathrm{min} / 100 \mathrm{ml}$ (IQR, 79.5; range, $23.4-160.8 \mathrm{ml} / \mathrm{min} / 100 \mathrm{ml}$ ) for SCL. Median HVPG was $19.5 \mathrm{mmHg}$ (IQR, 11; range $13-25 \mathrm{mmHg}$ ) in patients with portal thrombosis, and $14 \mathrm{mmHg}$ (IQR, 9; range, $2-28 \mathrm{mmHg}$ ) in those without portal thrombosis.

\section{Discussion}

Our study results showed a strong negative correlation between SCL and HVPG and a moderate negative correlation between SAF and HVPG. Using a SCL cut-off value of $125 \mathrm{ml} / \mathrm{min} / 100 \mathrm{ml}$ sensitivity for detection of severe $\mathrm{PH}$ $(\geq 12 \mathrm{mmHg}$ ) was $94 \%$, and specificity was $100 \%$. This indicates that perfusion CT of the spleen with calculation of the clearance which is the total flux of the contrast media from the intravascular space to the extravascular space in $\mathrm{ml} / \mathrm{min}$ per $100 \mathrm{ml}$ of tissue may be a non-invasive tool for estimation of the HVPG. We assume that a high portal venous pressure increases the pressure within the splenic interstitium which contradicts the flux of iodine from the intravascular space to the extravascular interstitial space. The correlation between SAF and HVPG is in accordance with the limited literature. In a study by Tsushima et al. [18], the authors reported a lower SAF in patients with chronic liver disease than in a control group and also found a significant negative correlation between SAF and WHVP in 11 patients. In contrast to our study, the correlation between SAF and WHVP was strong $(\mathrm{r}=0.741, \mathrm{p}=0.0024)$, whereas in our study the correlation between SAF and HVPG was moderate. However, another study [19] failed to show a statistically significant difference in SAF between patients with and patients without cirrhosis, although a trend toward lower perfusion in patients with cirrhosis was observed. In a study by Sauter et al. [20] on dynamic contrast-enhanced splenic CT in patients with and without cirrhosis, splenic BV and $\mathrm{K}$ (trans), which is a parameter used in different perfusion software and which is comparable to SCL, were statistically significant different between the two groups, which is for K(trans) in accordance with our study. However, no correlation with HVPG was performed in the study by Sauter et al. [20].

The liver perfusion parameters did not correlate with HVPG and failed to separate patients with severe PH from patients with moderate $\mathrm{PH}$. To the best of our knowledge, no data are available in the literature on hepatic CT perfusion in patients with $\mathrm{PH}$. However, several studies assessed perfusion with scintigraphy and a clear reduction of portal perfusion was shown in patients with $\mathrm{PH}$ compared to healthy patients [21-23]. The sensitivity of scintigraphy in detecting PH, based on a portal contribution of $\leq 66 \%$, was $62 \%$, and specificity was $100 \%$. Portal contribution to liver perfusion was statistically significantly negative correlated to HVPG $(\mathrm{r}=-0.43)$ [22]. We could not reproduce this finding in our CT perfusion study. Using contrast-enhanced ultrasonography, Jeong et al. [24] reported that the intrahepatic transit time, which is the time from hepatic arterial arrival to hepatic venous arrival, moderately correlated with HVPG $(r=-0.613)$ and that this parameter was the most accurate one to diagnose severe PH ( $\geq 12 \mathrm{mmHg})$. Using a cut-off value of $6 \mathrm{~s}$, sensitivity was $91 \%$ and $85 \%$, and specificity was $89 \%$ and $78 \%$, respectively, for the two reviewers. The false-positive rate was only $2 \%$; however, the false-negative rates were $35 \%$ and $40 \%$, respectively. With the software used in our study, the intrahepatic transit time was not available and this parameter could not be compared to the literature. In a recent study by Annet et al. [25] on hepatic flow parameters measured with MR imaging, HVPG was significantly correlated with all flow parameters. The correlation was substantial for the portal fraction $(\mathrm{r}=-0.769, \mathrm{p} \leq .001)$, the apparent portal perfusion $(\mathrm{r}=-0.726, \mathrm{p} \leq .001)$, and the mean transit time $(\mathrm{r}=0.721$, $\mathrm{p} \leq .001)$. Weak but significant correlations were found for apparent arterial perfusion $(\mathrm{r}=0.542, \mathrm{p} \leq .001)$, and distribution volume $(\mathrm{r}=0.437, \mathrm{p} \leq .002)$. In contrast, no significant association was found between HVPG and the portal flow in a study by Gouya et al. [26], whereas the azygos flow and HVPG were significantly correlated. The different imaging techniques with different models of perfusion calculation may contribute to the inconsistent results.

Splenic volume was not significantly correlated to HVPG in our study. This is in contrast to a study by Kihira et al. [27] which showed such a correlation. However, the correlation 
coefficient was only weak $(0.32)$ in that study. In a recent study by Pickhardt et al. [28] splenic volume allowed for non-invasive staging of hepatic fibrosis, but this finding may not be transferable to patients with cirrhosis and different levels of portal hypertension.

Using different ROI settings for separation between HAF and PVF led to significant differences in PVF and HPI calculation in our patients. This is in accordance with a study by Fischer et al. [16], which showed that peak splenic enhancement, which defines the break point between HAF and PVF, is delayed in patients with $\mathrm{PH}$, resulting in a miscalculation of hepatic perfusion parameters. The authors recommended using peak renal enhancement instead of peak splenic enhancement in patients with $\mathrm{PH}$. In our study, the break point was manually set at the crossing point of the aortal and portal venous TDC, which indicates the time point of replacement of the HAF by the PVF. We believe that this may be a more accurate method for hepatic blood flow separation than using a ROI within the renal parenchyma.

The small number of patients which may have influenced the results and overestimated sensitivity and specificity in detection of severe $\mathrm{PH}$ has to be considered as a limitation of this study. However, the standard error of the AUC in ROC analysis was only 0.04 and consequently a larger study cannot completely contradict our results. Only improvements of the estimates of the level of sensitivity and specificity will occur. Further studies are needed to confirm our first promising findings. The limitation of the results to the specific type of CT scanner, software, scan protocol and injection protocol used in this study has to be considered as further limitation of the study. Parameter values from our study cannot be transferred to $\mathrm{CT}$ perfusion using different models of perfusion calculation. In addition, other perfusion parameters such as mean transit time were not available in the used model and could not be assessed.

In conclusion, on CT-perfusion in patients with $\mathrm{PH}, \mathrm{SCL}$ showed a strong correlation with HVPG and may be used as a parameter for detection of severe $\mathrm{PH}$.

Acknowledgements Open access funding provided by Medical University of Graz. The scientific guarantor of this publication is Helmut Schoellnast, M.D. The authors of this manuscript declare relationships with the following company: Toshiba Medical Systems. Helmut Schoellnast, M.D. gave paid lectures in the past. The authors state that this work has not received any funding. One of the authors has significant statistical expertise. Institutional Review Board approval was obtained. Written informed consent was obtained from all subjects (patients) in this study. No study subjects or cohorts have been previously reported. Methodology: prospective, diagnostic study, performed at one institution.

Open Access This article is distributed under the terms of the Creative Commons Attribution 4.0 International License (http:// creativecommons.org/licenses/by/4.0/), which permits unrestricted use, distribution, and reproduction in any medium, provided you give appropriate credit to the original author(s) and the source, provide a link to the Creative Commons license, and indicate if changes were made.

\section{References}

1. Thabut D, Moreau R, Lebrec D (2011) Noninvasive assessment of portal hypertension in patients with cirrhosis. Hepatology 53:683694

2. Lebrec D, Sogni P, Vilgrain V (1997) Evaluation of patients with portal hypertension. Baillieres Clin Gastroenterol 11:221-241

3. Gadano A, Hadengue A, Vachiery F et al (1997) Relationship between hepatic blood flow, liver tests, haemodynamic values and clinical characteristics in patients with chronic liver disease. J Gastroenterol Hepatol 12:167-171

4. Garcia-Tsao G, Groszmann RJ, Fisher RL, Conn HO, Atterbury CE, Glickman M (1985) Portal pressure, presence of gastroesophageal varices and variceal bleeding. Hepatology 5:419-424

5. Lebrec D, De Fleury P, Rueff B, Nahum H, Benhamou JP (1980) Portal hypertension, size of esophageal varices, and risk of gastrointestinal bleeding in alcoholic cirrhosis. Gastroenterology 79: $1139-1144$

6. Groszmann RJ, Garcia-Tsao G, Bosch J et al (2005) Beta-blockers to prevent gastroesophageal varices in patients with cirrhosis. $\mathrm{N}$ Engl J Med 353:2254-2261

7. Ripoll C, Groszmann R, Garcia-Tsao G et al (2007) Hepatic venous pressure gradient predicts clinical decompensation in patients with compensated cirrhosis. Gastroenterology 133:481-488

8. Ripoll C, Groszmann RJ, Garcia-Tsao G et al (2009) Hepatic venous pressure gradient predicts development of hepatocellular carcinoma independently of severity of cirrhosis. J Hepatol 50:923-928

9. Merkel C, Bolognesi M, Bellon S et al (1992) Prognostic usefulness of hepatic vein catheterization in patients with cirrhosis and esophageal varices. Gastroenterology 102:973-979

10. Silva-Junior G, Baiges A, Turon F et al (2015) The prognostic value of hepatic venous pressure gradient in patients with cirrhosis is highly dependent on the accuracy of the technique. Hepatology 62:1584-1592

11. Guan S, Zhao WD, Zhou KR, Peng WJ, Mao J, Tang F (2005) CT perfusion at early stage of hepatic diffuse disease. World J Gastroenterol 11:3465-3467

12. Hashimoto K, Murakami T, Dono K et al (2006) Assessment of the severity of liver disease and fibrotic change: the usefulness of hepatic CT perfusion imaging. Oncol Rep 16:677-683

13. Van Beers BE, Leconte I, Materne R, Smith AM, Jamart J, Horsmans Y (2001) Hepatic perfusion parameters in chronic liver disease: dynamic CT measurements correlated with disease severity. AJR Am J Roentgenol 176:667-673

14. Fleischmann D, Chin AS, Molvin L, Wang J, Hallett R (2016) Computed Tomography Angiography: A Review and Technical Update. Radiol Clin North Am 54:1-12

15. Miles KA, Griffiths MR (2003) Perfusion CT: a worthwhile enhancement? Br J Radiol 76:220-231

16. Fischer MA, Brehmer K, Svensson A, Aspelin P, Brismar TB (2016) Renal versus splenic maximum slope based perfusion CT modelling in patients with portal-hypertension. Eur Radiol. doi:10. 1007/s00330-016-4277-7

17. Groszmann RJ, Wongcharatrawee S (2004) The hepatic venous pressure gradient: anything worth doing should be done right. Hepatology 39:280-282

18. Tsushima Y, Koizumi J, Yokoyama H, Takeda A, Kusano S (1998) Evaluation of portal pressure by splenic perfusion measurement using dynamic CT. AJR Am J Roentgenol 170:153-155

19. Blomley MJ, Kormano M, Coulden R, Lim-Dunham J, Dawson P, Lipton MJ (1997) Splenic blood flow: evaluation with computed tomography. Acad Radiol 4:13-20

20. Sauter AW, Spira D, Schulze M, Horger MS (2014) Explanations for the heterogeneity of splenic enhancement derived from blood 
flow kinetic measurements using dynamic contrast-enhanced CT (DCE-CT). Acta Radiol 55:645-653

21. Biersack HJ, Torres J, Thelen M, Monzon O, Winkler C (1981) Determination of liver and spleen perfusion by quantitative sequential scintigraphy: results in normal subjects and in patients with portal hypertension. Clin Nucl Med 6:218-220

22. Dao T, Elfadel S, Bouvard G et al (1993) Assessment of portal contribution to liver perfusion by quantitative sequential scintigraphy and Doppler ultrasound in alcoholic cirrhosis. Diagnostic value in the detection of portal hypertension. J Clin Gastroenterol 16:160-167

23. Gianpaolo M, Massimo B, Enzo S, Corrado M (1989) Assessment of liver circulation by quantitative scintiangiography: evaluation of the relative contribution of the hepatic arterial and portal venous blood flows to liver perfusion. Eur J Nucl Med 15:211-216

24. Jeong WK, Kim TY, Sohn JH, Kim Y, Kim J (2015) Severe portal hypertension in cirrhosis: evaluation of perfusion parameters with contrast-enhanced ultrasonography. PLoS One 10, e0121601
25. Annet L, Materne R, Danse E, Jamart J, Horsmans Y, Van Beers BE (2003) Hepatic flow parameters measured with MR imaging and Doppler US: correlations with degree of cirrhosis and portal hypertension. Radiology 229:409-414

26. Gouya H, Grabar S, Vignaux O et al (2016) Portal hypertension in patients with cirrhosis: indirect assessment of hepatic venous pressure gradient by measuring azygos flow with 2Dcine phase-contrast magnetic resonance imaging. Eur Radiol 26:1981-1990

27. Kihira S, Kagen AC, Vasudevan P et al (2016) Non-invasive prediction of portal pressures using CT and MRI in chronic liver disease. Abdom Radiol (NY) 41:42-49

28. Pickhardt PJ, Malecki K, Hunt OF et al (2016) Hepatosplenic volumetric assessment at MDCT for staging liver fibrosis. Eur Radiol. doi:10.1007/s00330-016-4648-0 